\title{
Range and Direction of Arrival Estimation of Near-Field Sources in Sensor Arrays using Differential Evolution Algorithm
}

\author{
Yawar Ali Sheikh \\ Department of Electronic \\ Engineering and Information \\ Science, USTC, Hefei, Anhui, \\ 230027 China
}

\author{
Rizwan Ullah \\ Department of Electronic \\ Engineering and Information \\ Science, USTC, Hefei, Anhui, \\ 230027 China
}

\author{
Zhongfu Ye \\ Department of Electronic \\ Engineering and Information \\ Science, USTC, Hefei, Anhui, \\ 230027 China
}

\begin{abstract}
Many algorithms have been proposed in recent years for source localization in near field. Some are based on subspace methods and some use evolutionary computing techniques. This article presents the performance of Differential Evolution (DE) algorithm for range and DOA estimation of near field narrow band sources, impinging on a uniform linear array (ULA) of passive sensors. Mean square error (MSE) is used as a fitness function because it requires only a single snapshot to converge and perform better even in negative SNR. The main contribution of this work is to explore the effectiveness of DE without hybridization for uniform linear arrays. The results of DE are compared with the results of Genetic Algorithm (GA). The effectiveness of both the algorithms is tested on the basis of a large number of Monte-Carlo simulations and their statistical analysis.
\end{abstract}

\section{Keywords}

Differential Evolution, Direction of Arrival, Evolutionary Computing, Near field, Source Localization.

\section{INTRODUCTION}

Source localization is an essential step in many fields of engineering e.g. array signal processing, speech signal processing, wireless communication etc. [1,2]. A number of Direction of Arrival (DOA) estimation algorithms, for far field narrow band sources, have been developed during the past years. The case becomes more intricate when the sources are in the Fresnel zone (near field) of array aperture [3]. The wave-front impinging on the array becomes spherical and sources cannot be localized by only estimating the DOAs. Range information is also needed to correctly localize the sources [4]. Near field case practically occurs in various situations like under water source localization, seismic exploration, speech enhancement with microphone arrays and ultrasonic imaging etc. [5]. Scholars from various parts of the world have proposed many approaches for near-field source localization, such as the high-order spectra (HOS) based algorithms, the two-dimensional (2D) Multiple Signal Classification (MUSIC) method, Estimation of Signal Parameters via Rotational Invariance Technique (ESPRIT) method, Maximum Likelihood (ML) method, the weighted linear prediction method and so on [6-11]. Most of these proposed techniques were computationally heavy in practical situations, some needed extra computations to pair the parameters in case of multiple, closely spaced sources which resulted in poor estimation in low Signal to Noise Ratio (SNR).
Evolutionary computing techniques such Genetic algorithm (GA), Particle Swarm Optimization (PSO), Genetic programming (GP) and Differential Evolution (DE) etc. have proved their significance in recent development period [12]. These techniques are easy to employ, powerful global optimizers and avoid getting stuck in local minima. Another attractive feature of these algorithms is they can be hybridized, for more reliability and effectiveness if needed, at the cost of additional computational complexity, with any efficient local optimizers such as Active set (AS), Interior point Algorithm (IPA), pattern Search (PS) etc. [13-17]. Among these global optimizers, DE is the fastest and easiest to employ. It even gives best performance without hybridization in some cases. Like GA, DE has also been successfully applied to a variety of problems.

This article presents the performance of DE for range and DOA estimation of near field narrow band sources impinging on a uniform linear array (ULA) of passive sensors. The core contribution of this work is to show the effectiveness and ease in application of DE to the problem of near field source localization without cascading with any local optimizers for optimum results. Mean square error (MSE) is used as a fitness evaluation function which is extensively used in literature. MSE is used because it requires only a single snapshot to converge and perform better even in negative SNR. The results of DE are compared with the results of GA. The effectiveness of both the algorithms is tested on the basis of large number of Monte-Carlo simulations and their statistical analysis.

The rest of the article is organized as follows: section 2 describes the data model; section 3 discusses the methodology employed. Section 4 is dedicated for results and discussion and section 5 gives conclusion and future work plan.

\section{DATA MODEL}

Consider a ULA consists of $M$ passive sensors. The interelement spacing between two consecutive sensors is equal and fixed to $d$ as shown in Fig. 1 . Sensor ' 0 ' is taken as the reference sensor. Suppose that $K$ narrow band signals in near field are 


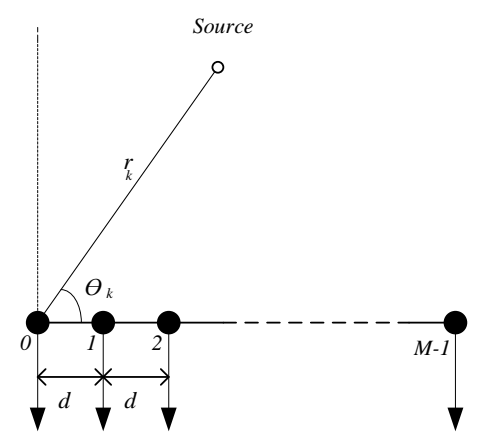

Fig. 1: Uniform linear array having $M$ passive sensors

impinging on the ULA from different directions. It is assumed that the number of sources is known. Alternately, the number of sources can be obtained by using methods like Akaike Information Theoretic Criteria (AIC) or Minimum Description Length (MDL). The incoming signals are assumed to have amplitude ' $s_{k}$ ', DOAs ' $\theta_{k}$ ' and ranges ' $r_{k}$ ' where $k=1,2, \ldots, K$.

It is assumed that $K \leq M$ and the amplitudes ' $s_{k}$ ' are taken same for all sensors. The same amplitude assumption is taken to make the simulations less complex and it is widely used in literature. It is also assumed that the incident signals and noise are uncorrelated. The angles ' $\theta_{k}$ ' are estimated w.r.t. the reference sensor ' 0 '. Using Fresnel approximation for near field sources, the signal received at $m^{\text {th }}$ sensor can be written as:

$$
\begin{array}{r}
x_{m}=\sum_{k=1}^{K} s_{k} e^{j\left(m \omega_{k}+m^{2} \emptyset_{k}\right)}+n_{m} ; \\
m=0,1, \ldots, M-1
\end{array}
$$

where,

$$
\omega_{k}=-\frac{2 \pi d}{\lambda} \sin \theta_{k}
$$

and

$$
\emptyset_{k}=\pi \frac{d^{2}}{\lambda r_{k}} \cos ^{2} \theta_{k}
$$

In vector form,

$$
\boldsymbol{x}=\boldsymbol{A}(\theta, r) \boldsymbol{s}+\boldsymbol{n}
$$

where,

$$
\begin{aligned}
& \boldsymbol{x}=\left[x_{0}, x_{1}, \ldots, x_{M-1}\right]^{T} \\
& \boldsymbol{s}=\left[s_{1}, s_{2}, \ldots, s_{K}\right]^{T} \\
& \boldsymbol{A}(\theta, r)=\left[\boldsymbol{a}\left(\theta_{1}, r_{1}\right), \boldsymbol{a}\left(\theta_{2}, r_{2}\right), \ldots, \boldsymbol{a}\left(\theta_{K}, r_{K}\right)\right] \\
& \boldsymbol{n}=\left[n_{0}, n_{1}, \ldots, n_{M-1}\right]^{T} \\
& \boldsymbol{a}\left(\theta_{k}, r_{k}\right) \\
& =\left[1, e^{j\left(\omega_{k}+\emptyset_{k}\right)}, e^{j\left(2 \omega_{k}+2^{2} \emptyset_{k}\right)}, \ldots, e^{j\left((M-1) \omega_{k}+(M-1)^{2} \emptyset_{k}\right)}\right]^{T}
\end{aligned}
$$

for $k=1,2, \ldots, K \cdot \boldsymbol{a}\left(\theta_{k}, r_{k}\right)$ is the steering vector and $\boldsymbol{n}$ is the additive white noise at the output of the array. It is clear that the problem in hand is to estimate the unknown parameters i.e. the angle of arrival ' $\theta_{k}$ ' and the range ' $r_{k}$ ' of sources, for $k=1,2, \ldots, K$ from the received data.

\section{THE METHOGOLOGY}

\subsection{Differential Evolution Algorithm}

Differential Evolution is a mathematical prototype of complex natural process of evolution. It is a stochastic, populationbased optimization algorithm which is one of the most powerful global optimization tools. The flow diagram of DE is shown in Fig. 2. It is based on three operators [18],
a. Mutation
b. Cross-over
c. Selection

and three parameters:
d. Population size
e. Scale factor
f. Cross-over rate (or probability)

The basic steps for DE algorithm are:

Step 1 Initialization: Suppose that $\mathrm{L}$ and $\mathrm{H}$ are the lower and upper limits of chromosomes respectively. Suppose that the number of chromosomes in a generation is ' $\mathrm{C}$ ' and number of genes in any chromosome is ' $\mathrm{G}$ ', then

$$
\bar{x}_{g e}^{c, g}=L+\operatorname{rand}() *(H-L)
$$

where,

$c=$ chromosome number for $1 \leq i \leq \mathrm{C}$

$g e=$ gene number for $1 \leq g e \leq \mathrm{G}$

$g=$ generation number

$\operatorname{rand}()=$ random number chosen from 0 to 1

Step 2 Updating: Update all the chromosomes of the present generation ' $g$ ' from 1 to C. Suppose we pick up $c^{\text {th }}$ chromosome $\overline{\boldsymbol{x}}^{c, g}$.

\section{a) Mutation:}

Choose three numbers randomly from 1 to $\mathrm{C}$ i.e. $\left(c_{1}, c_{2}, c_{3}\right)$, all different and not equal to $c$.

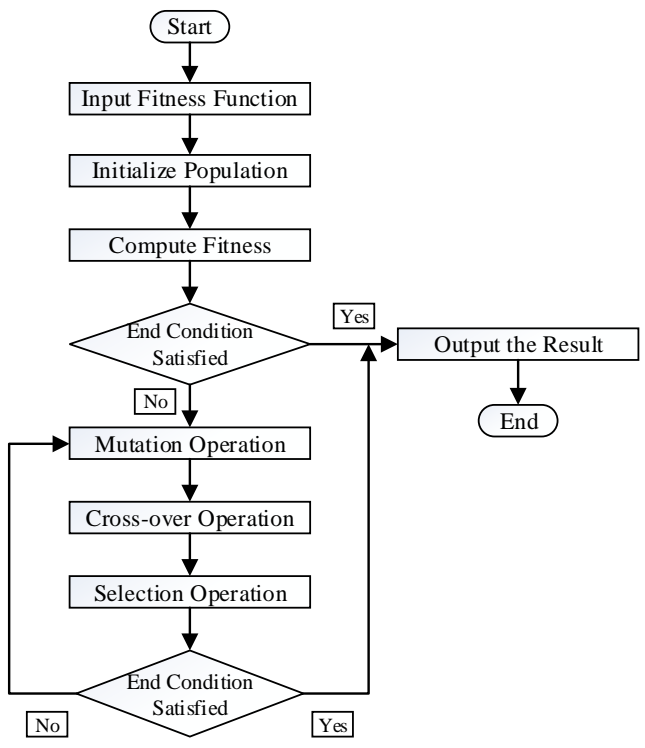

Fig. 2: Flow diagram of DE Algorithm

$$
\overline{\boldsymbol{y}}^{c, g+1}=\overline{\boldsymbol{x}}^{c_{1}, g}+F\left(\overline{\boldsymbol{x}}^{c_{2}, g}-\overline{\boldsymbol{x}}^{c_{3}, g}\right)
$$

where, ' $F$ ' is the scale factor (problem dependent) which can be selected from 0.4 to 1.2 but usually selected as 0.5 . 


\section{b) Crossover:}

$$
\bar{z}_{g e}^{c, g+1}=\left\{\begin{array}{ll}
\bar{y}_{g e}^{c, g+1} & \text { if rand }() \leq C R \text { or ge }=g e_{\text {rand }} \\
\bar{x}_{g e}^{c, g} & \text { otherwise }
\end{array}\right\} \forall j^{\prime} s
$$

where, rand ( ) is a number randomly selected from 0 to 1 , $C R$ (cross-over rate) $=0.5$ to 0.9 (generally) and $g e_{\text {rand }}$ is chosen randomly from 1 to $\mathrm{G}$.

c) Selection:

$$
\overline{\boldsymbol{x}}^{c, g+1}=\left\{\begin{array}{ll}
\overline{\boldsymbol{z}}^{c, g+1} & \text { if } f\left(\overline{\boldsymbol{z}}^{c, g+1}\right)<f\left(\overline{\boldsymbol{x}}^{c, g}\right) \\
\overline{\boldsymbol{x}}^{c, g} & \text { otherwise }
\end{array}\right\}
$$

where,

$$
f\left(\overline{\boldsymbol{x}}^{c}\right)=\left\|\overline{\boldsymbol{x}}-\overline{\boldsymbol{x}}^{c}\right\|^{2}
$$

is the mean square error. Repeat the above steps for all chromosomes.

\section{Step 3 Termination: If}

$$
f\left(\overline{\boldsymbol{x}}^{c, g+1}\right)<\varepsilon
$$

' $\varepsilon$ ' is a very small positive number, or if the number of generations reached, stop the procedure otherwise go back to step 2.

The above steps of DE are applied to near field source localization problem as follows:

First, create the initial generation of chromosomes randomly by using (3). The chromosomes consists of genes in the following order:

$$
\overline{\boldsymbol{x}}^{c, g}=\left[\begin{array}{c}
r_{1} \\
\vdots \\
r_{K} \\
\theta_{1} \\
\vdots \\
\theta_{K}
\end{array}\right]
$$

After creating the generation of initial chromosomes, the algorithm checks the fitness of all the chromosomes by using (7), where $\overline{\boldsymbol{x}}^{c}$ is calculated by using $\overline{\boldsymbol{x}}^{c, g}$ in (2). If any of the chromosomes satisfies the condition of termination, the algorithm stops. Otherwise, it goes to the updating process.

\section{RESULTS AND DISCUSSION}

This section discusses the performance of DE algorithm for DOA and range estimation of near field sources in the presence of noise. Noise is considered as white Gaussian for all cases. A uniform linear array of 20 passive sensors is used. The spacing between two consecutive sensors is taken as 0.5 $\lambda$. It is assumed that the number of sources $K$ is known. The results of proposed algorithm are compared with the results of GA for DOA and range estimation of near field sources in the presence of noise. Both the algorithms are tested on the basis of changing SNR, different number of sources and different number of array sensors used. The results are averaged over 100 Monte-Carlo simulations.

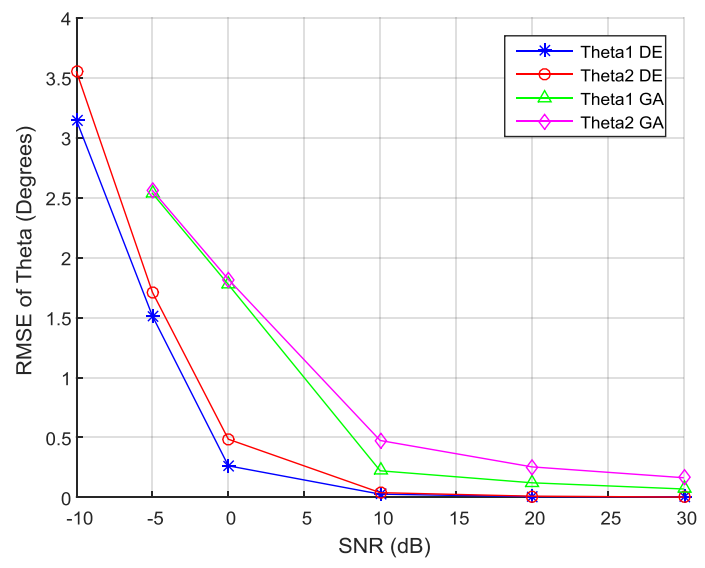

Fig. 3: RMSE of DOA vs SNR for DE and GA

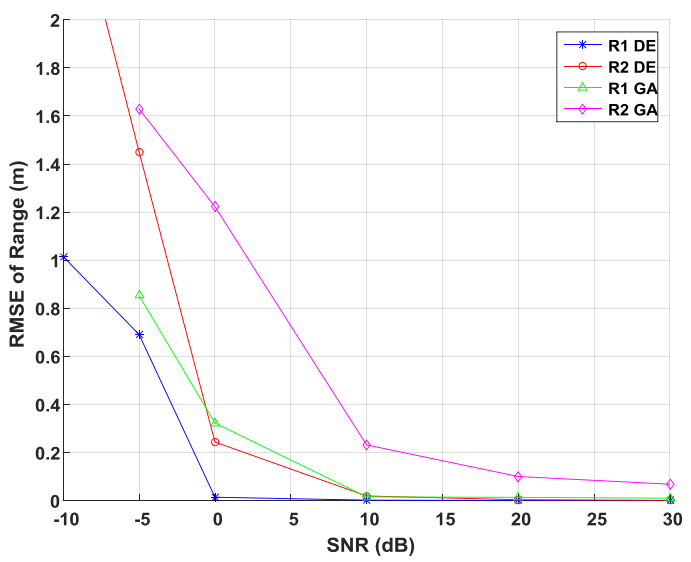

Fig. 4: RMSE of Range vs SNR for DE and GA

Case 1: This case presents the performance of $D E$ for changing SNR from $-10 \mathrm{~dB}$ to $30 \mathrm{~dB}$. For this, $M$ is taken as 20 , two narrow band sources coming from angles $\left(10^{\circ}, 40^{\circ}\right)$ are considered and source ranges are taken as $(6 \mathrm{~m}, 8 \mathrm{~m})$. For DE, 50 chromosomes and 100 generations are considered with $F=0.4, C R=0.8$ and $\lambda=1 \mathrm{~m}$. For GA, population size is taken 100 .

Form Fig. 3 and Fig. 4, it is clear that DE can perform more accurately than GA to estimate the DOAs and ranges. Even in negative SNR, DE can perform with some error but GA cannot detect the DOAs and ranges in case of SNR less than $5 \mathrm{~dB}$ and its performance is poorly effected.

Case 2: This case presents the performance of $D E$ for different number of sources. For this, $M$ is taken as 20 . Two, three and four sources are considered coming from angles $\left(10^{\circ}, 40^{\circ}\right),\left(20^{\circ}, 40^{\circ}, 60^{\circ}\right)$ and $\left(10^{\circ}, 20^{\circ}, 30^{\circ}, 40^{\circ}\right)$ and source ranges are taken as $(6 \mathrm{~m}, 8 \mathrm{~m}),(6 \mathrm{~m}, 8 \mathrm{~m}, 10 \mathrm{~m}),(6 \mathrm{~m}, 7 \mathrm{~m}, 8 \mathrm{~m}$, $9 \mathrm{~m}$ ) for two, three and four sources respectively. SNR is fixed at $20 \mathrm{~dB}$. For DE, 50 chromosomes and 100 generations are considered with $F=0.4, C R=0.8$ and $\lambda=1 \mathrm{~m}$. For GA, population size is taken 200 .

Fig. 5 and Fig. 6 shows the performance of DE for DOA and range estimation of two, three and four sources. It is clear that DE performed very well and much better than GA in all cases. DE faced some local minima in case of four sources and its 
performance is slightly effected for range estimation but still it gave better results than GA.

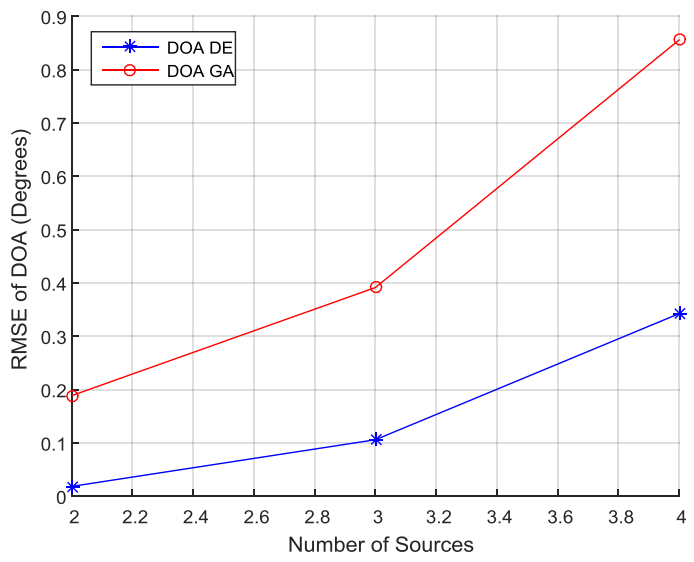

Fig. 5: Joint RMSE of Theta vs ' $K$ ' for DE and GA

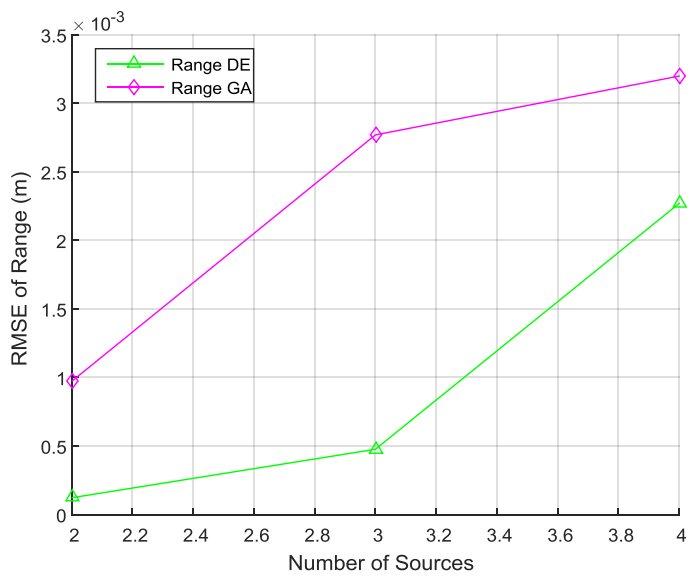

Fig. 6: Joint RMSE of Range vs ' $K$ ' for DE and GA

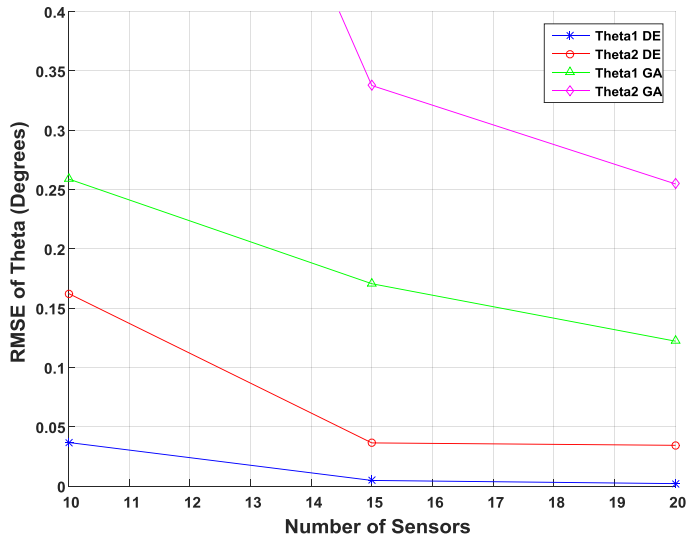

Fig. 7: RMSE of DOA vs ' $M$ ' for DE and GA

Case 3: This case presents the performance of DE for different number of array sensors used. Two sources are considered coming from angles $\left(10^{\circ}, 40^{\circ}\right)$ and source ranges are taken as $(6 \mathrm{~m}, 8 \mathrm{~m})$. SNR is fixed at $20 \mathrm{~dB}$. For DE, 50 chromosomes and 100 generations are considered with $F=$ $0.4, C R=0.8$ and $\lambda=1 \mathrm{~m}$. For GA, population size is taken 100.
Fig. 7 and Fig. 8 shows the performance of DE for DOA and range estimation of two sources when 10,15 and 20 array

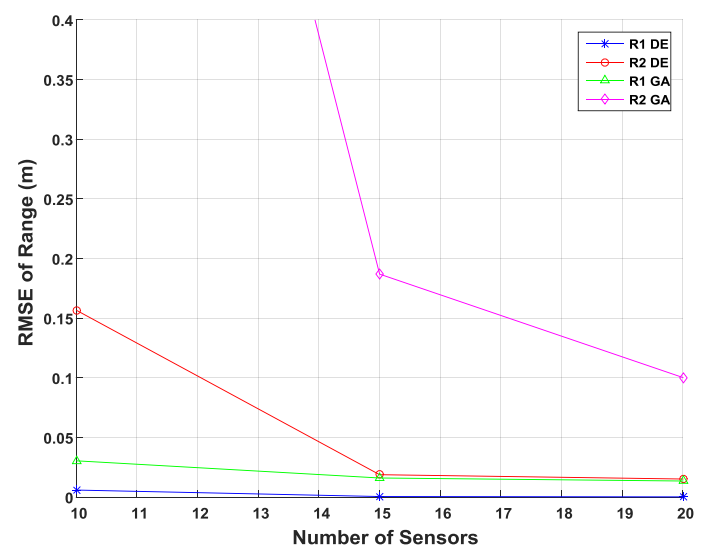

Fig. 8: RMSE of Range vs ' $M$ ' for DE and GA

sensors are used. It is clear that DE performed very accurately and better than GA in all cases. Both the algorithms can work when the number of array sensors is greater than or equal to the number of sources. Also, by increasing the number of array sensors, the accuracy of both algorithms increases.

\section{CONCLUSION AND FUTURE PLAN}

In this work, $\mathrm{DE}$ has been employed without hybridization for range and DOA estimation of near field narrow band sources impinging on a uniform linear array of passive sensors. Mean square error is used as a fitness function because it requires only a single snapshot to converge. From a large number of simulations, it has been proved that DE has produced better results as compared to GA in terms of robustness to noise, convergence rate and estimation accuracy. The proposed algorithm fails when the number of sensors in the ULA is less than the number of sources as it becomes an underdetermined case.

In future, this technique can be applied for wide band source localization in speech signal processing.

\section{REFERENCES}

[1] Byrne, D., O'Halloran, M., Glavin, M., Jones, E. 2010. Data independent radar beam forming algorithms for breast cancer detection. PIER 107, 331-348.

[2] Nishiura, T., Nakamura. 2003. Talker localization based on the combination of DOA estimation and statistical sound source identification with microphone array. In IEEE Workshop Statistical Signal Processing, 597-600.

[3] Zaman, F., Qureshi, I. M., Naveed, A., Khan, Z. U. 2012. Joint Estimation of Amplitude, Direction of Arrival and Range of Near Field Sources using Memetic Computing. PIER C, 31, 199-213.

[4] Liang, J., Liu, D., Zeng, X., Wang, W., Zhang, J., Chen, H. 2011. Joint (azimuth-elevation-range) estimation of mixed near-field and far-field sources using two-stage separated steering vector-based algorithm. PIER, 113, $17-46$.

[5] Kim, J. H., Yang, I. S., Kim, K. M., Oh, W. T. 2000 Passive ranging sonar based on multi-beam towed array. In Proc. IEEE Oceans, 3, 1495-1499. 
[6] Raghu, N. C., Sanyogita, S. 1995. Higher-order subspace based algorithms for passive localization of near-field sources. In Proc. Twenty-Ninth Asilomar Conference Signals, Systems and Computers, Pacific Grove, CA, 777-781.

[7] Huang, Y. D., Barkat, M. 1991. Near-field multiple sources localization by passive sensor array. IEEE Trans. Antennas Propag., 39(7): 968-975.

[8] Y. Zhou, D. Feng, "A new subspace method for the estimation of parameters of near field sources", Journal of Xidian Univ., 2006, 39(5): 41-45.

[9] Diao, M., Miao, S. 2001. New method of parameter matching for 2-D ESPRIT algorithms. Syst. Eng. Electron, 29(8): 1126-1129.

[10] Ziskind, I., Wax, M. 1988. Maximum likelihood localization of multiple sources by alternating projection. IEEE Trans. on Acoust., Speech, Signal Processing, 36, No. 10, 1553-1560.

[11] Abred-Meraim, K., Hua, Y. 1998. 3-D near field source localization using second order statistics. In Conf. Record of the 31st Asilomar Conf. on Signals, Systems and Computers, Pacific Grove, CA, USA, 1307-1311.

[12] Sheikh, Y. A., Zaman, F., Qureshi, I. M., Atique-urRehman, M. 2012. Amplitude and Direction of Arrival Estimation using Differential Evolution. In International Conference on Emerging Technologies, DOI: 10.1109/ICET.2012.6375456

[13] F. Zaman, I. M. Qureshi, A. Naveed, Z. U. Khan, "An Application of Artificial Intelligence for the Joint Estimation of Amplitude and Two Dimensional Direction of Arrival of far field sources using 2-L shape array", International Journal of Antennas and Propagation, 2013, Article ID 593247, 10 pages.

[14] Zaman, F., Qureshi, I. M., Naveed, A., Khan, J. A., Zahoor, R. M. A. 2012. Amplitude and Directional of Arrival Estimation: Comparison between different techniques. Progress in Electromagnetic research-B (PIER-B), 39, 319-335.
[15] F. Zaman, I. M. Qureshi, A. Naveed, Z. U. Khan, "Real Time Direction of Arrival estimation in Noisy Environment using Particle Swarm Optimization with single snapshot", Research Journal of Engineering and Technology (Maxwell Scientific organization), 2012, 4(13): 1949-1952.

[16] Zaman, F., Khan, J. A., Khan, Z. U., Qureshi, I. M. 2013. An application of hybrid computing to estimate jointly the amplitude and Direction of Arrival with single snapshot. In IEEE 10th IBCAST, 364-368.

[17] Zaman, F., Khan, S. U., Ashraf, K., Qureshi, I. M. 2014. An Application of Hybrid Differential Evolution to 3-D Near field Source localization. In Proceedings of 2014 11th International Bhurban Conference on Applied Sciences \& Technology (IBCAST).

[18] Ao, Y., Chi, H. 2009. Experimental Study on Differential Evolution Strategies. In Global Congress on Intelligent Systems, IEEE computer society, DOI 10.1109/GCIS.2009.31.

[19] Maulik, U. 2011. Analysis of gene microarray data in a soft computing framework. Engineering Applications of Artificial Intelligence, Elsevier, Signal Process, 24, 485490.

[20] R. Storn, K. Price, "Differential Evolution - A Simple and Efficient Heuristic for Global Optimization over Continuous Spaces”, Journal of Global optimization, 1997, 11: 341-359.

[21] Errastil, B., Escot, D., Poyatos, D., Montiel, I. 2009. Performance analysis of the Particle Swarm Optimization algorithm when applied to direction of arrival estimation. In ICEAA, 447-450.

[22] Addad, B., Amari, S., Lesage, J. 2011. Genetic algorithms for delays evaluation in networked automation systems. Engineering Applications of Artificial Intelligence, Elsevier, 24: 485-490.

[23] Jiankui, Z., Zishu, H., Benyong, L. 2006. Maximum Likelihood DOA Estimation Using Particle Swarm Optimization Algorithm. Proc. IEEE. 